
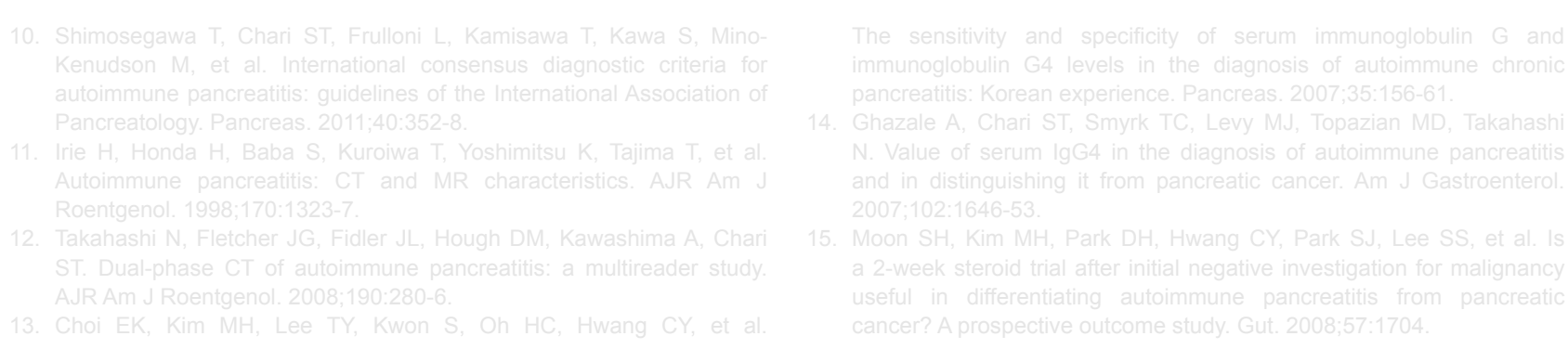

\title{
Miocardiopatia de Takotsubo: Na Origem do Choque Cardiogénico
}

\author{
Takotsubo Cardiomyopathy: Cause of a Cardiogenic Shock
}

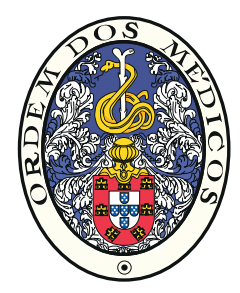

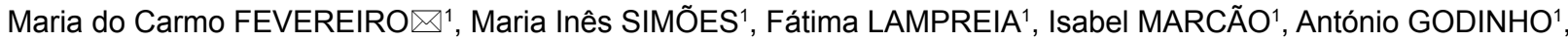 \\ Vítor LOPES ${ }^{2}$ \\ Acta Med Port 2015 Sep-Oct;28(5):670-673
}

\section{RESUMO}

A miocardiopatia de Takotsubo, de etiologia desconhecida, caracteriza-se pela disfunção sistólica súbita e transitória dos segmentos médio-apicais do ventrículo esquerdo, sem doença coronária significativa, com total normalização das alterações segmentares. É mais frequente em mulheres de meia-idade, implicando diagnóstico diferencial com a sindrome coronária aguda. Apresentamos o caso de uma mulher de 59 anos que recorreu ao Serviço de Urgência por dispneia súbita e dor torácica. À admissão apresentava-se em edema pulmonar agudo hipotensivo com necessidade de suporte aminérgico e ventilação invasiva. A avaliação analítica demonstrava elevação dos marcadores cardíacos. Electrocardiogramas seriados em ritmo sinusal com inversão progressiva da onda $T$ nas derivações precordiais (v2 - v6). Ecocardiogramas de controlo revelando acinésia apical com diminuição da função sistólica global, e reversão total das alterações em duas semanas. Admitido choque cardiogénico de etiologia não esclarecida foi excluída doença coronária, sustentando o diagnóstico de miocardiopatia de Takotsubo.

Palavras-chave: Choque Cardiogénico; Miocardiopatia de Takotsubo.

\section{ABSTRACT}

Takotsubo cardiomyopathy, of unknown etiology, is characterized by sudden and transient systolic dysfunction of the mid-apical segments of the left ventricle without significant coronary disease, and full normalization of segmental changes. More common in middle-aged women, it is cause of differential diagnosis with acute coronary syndrome. We present the case of a 59 year old woman admitted to the emergency room with sudden chest pain and dyspnea. At presentation: acute hypotensive pulmonary edema requiring aminergic support and invasive ventilation. Blood tests showed elevated necrosis myocardial enzymes. Serial electrocardiograms: sinus rhythm with progressive inversion of the T wave through the precordial leads (v2 - v6). Control echocardiograms: overall decreased systolic function with apical akinesia, and full reversal of the changes in 2 weeks. Cardiogenic shock of unknown etiology was admitted and a coronary computed tomography angiography was performed excluding coronary heart disease, supporting the diagnosis of Takotsubo cardiomyopathy.

Keywords: Shock, Cardiogenic; Takotsubo Cardiomyopathy.

\section{INTRODUÇÃO}

A miocardiopatia de Takotsubo (MCT), também chamada miocardiopatia de stress, síndrome do balonamento apical, acinésia/discinésia apical transitória do ventrículo esquerdo (VE) ou síndrome de broken heart, é uma entidade caracterizada pela disfunção sistólica súbita e transitória dos segmentos médio-apicais do VE, sem doença coronária significativa, e com total normalização das alterações segmentares. ${ }^{1-5}$

Mais frequente em mulheres, 80 - 100\% dos casos ocorre em idades entre 61 - 76 anos.

Apesar da etiopatogenese não estar totalmente escla- recida, são postulados mecanismos que incluem excesso de catecolaminas, espasmo coronário, isquémia microvascular ou obstrução aguda e dinâmica do tracto de saída do VE. ${ }^{3-6}$

Geralmente ocorre após situações de stress físico/ emocional, doença aguda ou utilizadores de drogas ilícitas, ${ }^{3,6}$ sendo a forma de apresentação mais frequente semelhante à síndrome coronário agudo (SCA), resultando numa prevalência estimada de $1,7-2,2 \%$ dos casos suspeitos de SCA. ${ }^{4,5,7,8}$

Os sintomas mais comuns são dor retrosternal súbita,

1. Unidade Funcional de Medicina 1.4. Hospital de São José. Centro Hospitalar de Lisboa Central. Lisboa. Portugal.

2. Unidade de Cuidados Intensivos Polivalente 1. Hospital de São José. Centro Hospitalar de Lisboa Central. Lisboa. Portugal.

$\triangle$ Autor correspondente: Maria do Carmo Fevereiro. carmofevereiro@yahoo.com

Recebido: 08 de Maio de 2015 - Aceite: 07 de Setembro de 2015 | Copyright @ Ordem dos Médicos 2015 
dispneia, palpitações, diaforese e síncope.

Como resultado da disfunção VE transitória podem desenvolver-se várias complicações nomeadamente insuficiência cardíaca, arritmias, instabilidade hemodinâmica com hipotensão e choque cardiogénico. ${ }^{5,7-9}$

Perante a ausência de critérios diagnósticos definitivos, considera-se MCT quando estão presentes quatro critérios modificados propostos pela Clínica Mayo: 1) hipocinésia, acinésia/discinésia transitória dos segmentos médios e apicais do VE com ou sem envolvimento apical, com alterações da contractilidade na área de vascularização correspondente a mais do que uma artéria coronária; 2) ausência de doença coronária obstrutiva ou evidência angiográfica de rutura de placa; 3) alterações electrocardiográficas de novo (supradesnivelamento de ST e/ou inversão da onda T) ou elevação da troponina; 4) ausência de feocromocitoma ou miocardite. ${ }^{1}$.

Os autores apresentam um caso ilustrativo desta entidade, cuja forma de apresentação foi a de choque cardiogénico.

\section{CASO CLÍNICO}

Mulher, 59 anos, com antecedentes de hipertensão arterial, dislipidémia e doença bipolar, recorreu ao Serviço de Urgência por dispneia súbita e dor torácica intensa, sem irradiação e sem factor desencadeante. À admissão apresentava-se em edema pulmonar agudo hipotensivo com necessidade de suporte aminérgico e ventilação invasiva. A avaliação laboratorial revelava leucocitose neutrofílica $\left(18,5 \times 10^{\wedge} \mathrm{g} / \mathrm{L}, 88 \% \mathrm{~N}\right)$, PCR 184 mg/L, disfunção

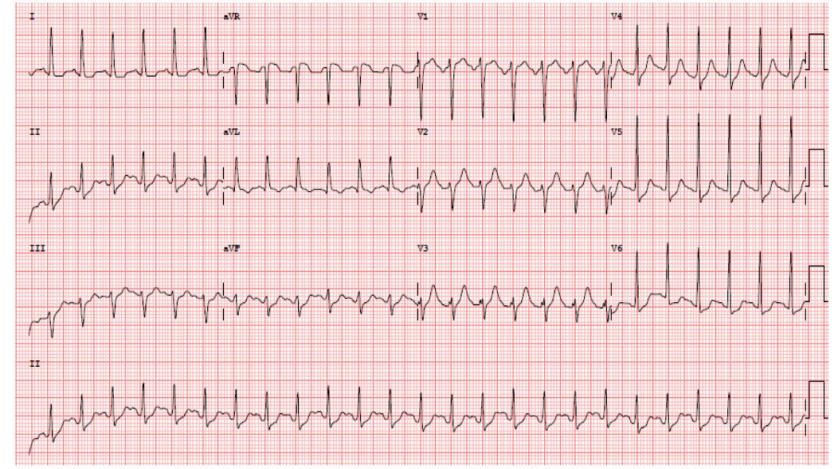

Figura 1 - Electrocardiograma seriado com inversão progressiva da onda T nas derivações précordiais v2 - v6

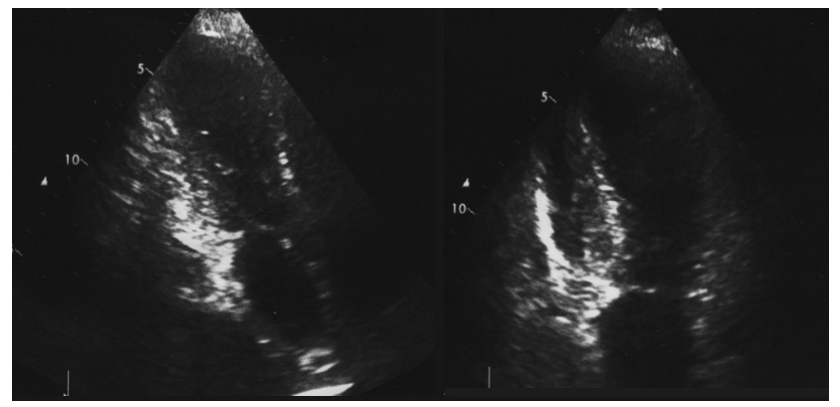

Figura 3 - Ecocardiograma bidimensional transtorácico, plano apical. Avaliação inicial: balonamento apical, hipocinésia do segmento mediano da parede inferior e anterior; hipercontractibilidade dos segmentos basais. renal (creatinina 1,22 mg/dL), LDH 402 U/L, elevação (estável) dos marcadores cardíacos (CK $300 \mathrm{U} / \mathrm{L}$, mioglobina $105 \mathrm{ng} / \mathrm{ml}$ e troponina I 0,98 ng/ml) e BNP $5000 \mathrm{pg} / \mathrm{ml}$. A teleradiografia de tórax mostrava cardiomegália e edema intersticial bilateral. Realizou electrocardiogramas seriados (Fig.s 1 e 2) que revelaram ritmo sinusal com inversão progressiva da onda $T$ nas derivações precordiais. O estudo microbiológico (hemocultura, urocultura, antigenúria para Pneumococcus e Legionella, serologias para Chlamydia, Mycoplasma e vírus respiratórios) foi negativo. O ecocardiograma revelou balonamento apical com hipocinésia do segmento mediano da parede inferior e anterior, e função sistólica global diminuída (Fig. 3). Durante o internamento, após desmame ventilatório e aminérgico, assistiu-se a meIhoria clínica com normalização dos marcadores cardíacos, das alterações electrocardiográficas e ecocardiográficas, com retorno a uma morfologia e função ventricular normais em duas semanas (Fig. 4).

Admitido choque cardiogénico de etiologia não esclarecida realizou angiotomografia coronária (Fig. 5) que excluiu doença coronária, sustentando o diagnóstico de MCT.

Após a alta manteve seguimento em consulta de medicina interna, permanecendo assintomática, com exames complementares normais, concordante com o bom prognóstico desta entidade justificado pela sua reversibilidade total.

\section{DISCUSSÃO}

A MCT é uma entidade clínica rara, cuja forma de apresentação é inespecífica e semelhante ao SCA. Exige

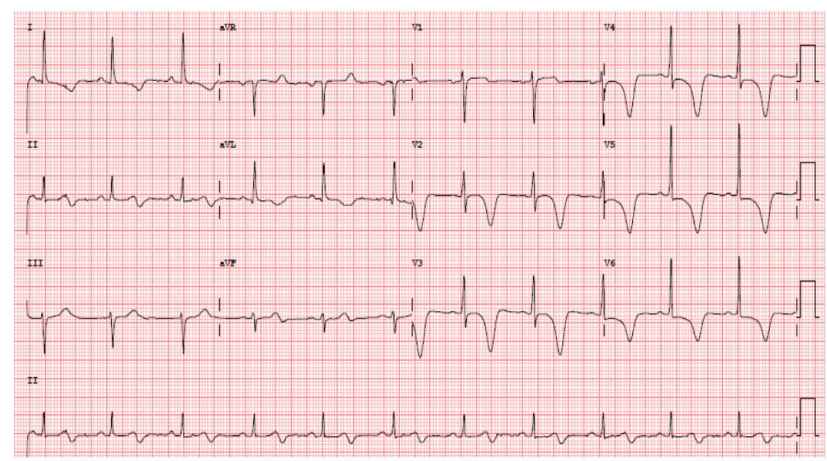

Figura 2 - Electrocardiograma seriado com inversão progressiva da onda T nas derivações précordiais v2 - v6

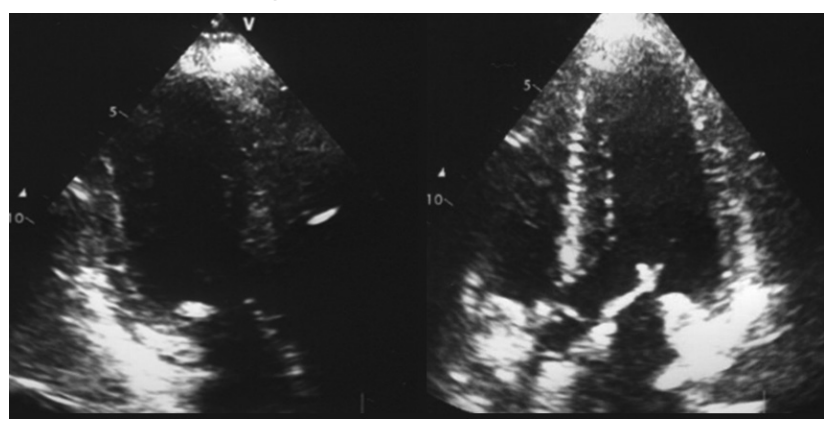

Figura 4 - Ecocardiograma bidimensional transtorácico, plano apical. Avaliação às 2 semanas: normalização da cinética segmentar do VE, função sistólica global conservada. 

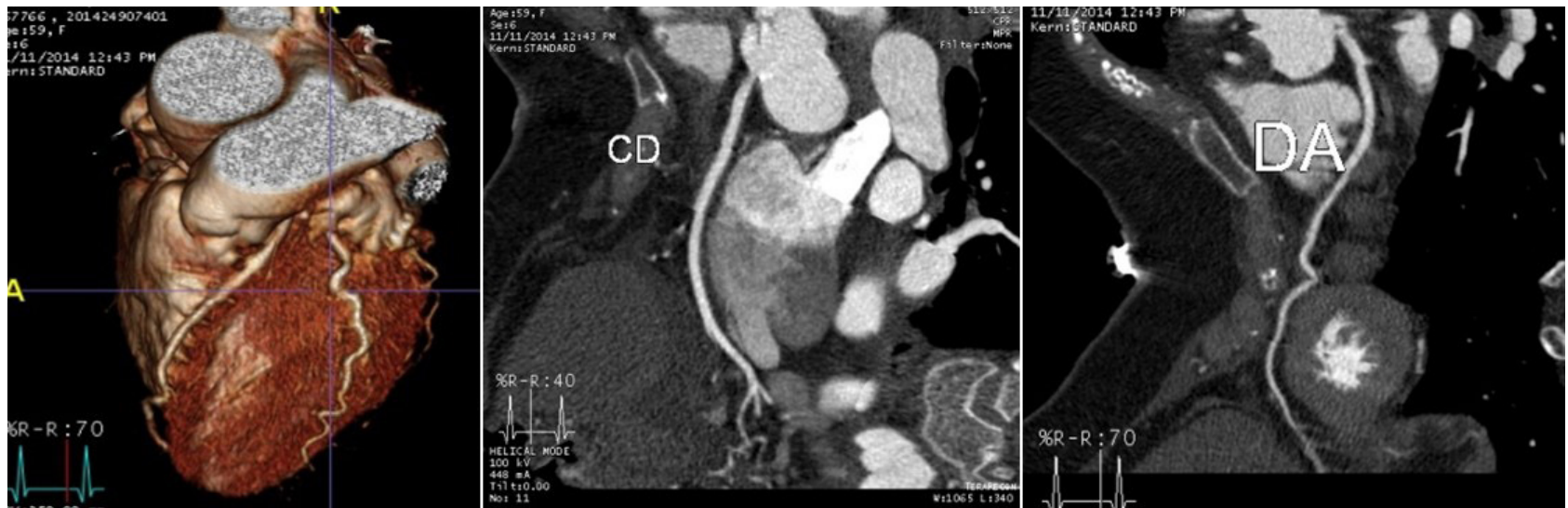

Figura 5-Angiotomografia computorizada coronária com coronárias normais; score de cálcio = 0, boa função sistólica global e segmentar $($ Vol TD $=81 \mathrm{ml} ; \mathrm{FEj}=81 \%)$.

elevada suspeição clínica e implementação de uma marcha diagnóstica rápida, nomeadamente na abordagem diferencial com o EAM com supradesnivelamento de ST, cuja terapêutica de eleição é distinta.

No que respeita aos exames complementares, cursa com alterações electrocardiográficas que à admissão podem ser normais / inespecíficas (15\%) ou mimetizar as de um SCA através da elevação do segmento ST (67 - 75\%), inversão da onda $\mathrm{T}(61 \%)$, ondas $Q$ ou progressão anormal das ondas R $(6-31 \%) .^{2}$ Os marcadores cardíacos (troponinas I e T) estão elevados em $90 \%$ dos casos, apesar de em menor grau comparativamente com os casos de EAM com supra-ST. ${ }^{1-4}$ Também o BNP aumenta, com valores superiores aos encontrados no EAM com supra-ST. ${ }^{10,11}$

Neste caso, a doente apresentou marcada discrepância entre uma disfunção cardíaca grave com uma pequena elevação dos marcadores cardíacos. O ecocardiograma revela acinésia/discinésia dos segmentos médios e apicais do VE com zona de acinésia correspondente ao território de perfusão de mais do que uma artéria coronária. A fracção de ejecção média do VE ronda $20-49 \% .^{2}$ O diagnóstico confirma-se por angiografia coronária na ausência de doença coronária.

Quanto à terapêutica, dada a reversibilidade implícita e incerteza fisiopatológica, baseia-se em medidas de suporte dirigidas às complicações agudas (que ocorrem em cerca de $20 \%$ dos casos), neste caso demonstrada pelo choque cardiogénico. ${ }^{2,5}$

Perante o choque, excluída obstrução aguda/dinâmica do tracto de saída do VE, instituem-se inotrópicos como a dobutamina. O papel dos simpaticomiméticos permanece por esclarecer, atendendo ao excesso catecolaminérgico como factor etiológico potencial. ${ }^{8}$

No caso, a terapêutica incluiu suporte hemodinâmico, inibidores da enzima de conversão da angiotensina e $\beta$-bloqueantes com importância demonstrada na remodelagem do VE e consequente melhoria de função.

A MCT cursa, geralmente, com uma evolução natural benigna, com remissão total das alterações morfológicas e funcionais em 2 - 4 semanas, sendo a sobrevida a longo prazo semelhante à da população geral. Contudo, encontra-se mencionada a possibilidade de recorrência desta patologia em $<10 \%$, com uma mortalidade intra-hospitalar inferior a $2 \% .{ }^{1,2,10,12}$

\section{CONCLUSÃO}

A MCT é uma entidade rara, em crescente reconhecimento a nível mundial, cuja etiologia e fisiopatologia permanecem por esclarecer. A apresentação clínica é inespecífica e impõe diagnóstico diferencial com SCA, sendo a suspeição clínica bem como a disponibilidade e realização atempada dos meios complementares de diagnóstico imprescindíveis. Salienta-se a importância da ecocardiografia que põe em evidência as alterações morfológicas e funcionais cardíacas transitórias, e a angiografia que comprova a ausência de doença coronária. Ao contrário do SCA, não tem tratamento específico, o prognóstico é favorável, sendo a insuficiência cardíaca a complicação mais frequente podendo ocorrer em $45 \%$ dos casos. ${ }^{10-12}$

\section{PROTECÇÃO DE PESSOAS E ANIMAIS}

Os autores declaram que os procedimentos seguidos estavam de acordo com os regulamentos estabelecidos pelos responsáveis da Comissão de Investigação Clínica e Ética e de acordo com a Declaração de Helsínquia da Associação Médica Mundial.

\section{CONFIDENCIALIDADE DOS DADOS}

Os autores declaram ter seguido os protocolos do seu centro de trabalho acerca da publicação dos dados de doentes.

\section{CONFLITOS DE INTERESSE}

Os autores declaram a inexistência de conflitos de interesse relativamente ao presente artigo.

\section{FONTES DE FINANCIAMENTO}

$O$ trabalho foi realizado sem o suporte de qualquer bolsa ou outro tipo de apoio financeiro. 


\section{REFERÊNCIAS}

1. Reeder G, Prasad A. Stress-induced (takotsubo) cardiomyopathy. UpToDate. 2014. [Consultado 2015 Jan 20]. Disponível em: http://www. uptodate.com/contents/stress-takotsubo-cardiomyopathy.

2. Tomich E, Luerssen E, Kang C. Takotsubo Cardiomyopathy. eMedicine Article. 2012. [Consultado 2015 Jan 20]. Disponível em: http:// emedicine.medscape.com/article/1513631-overview.

3. Gianni M, Dentali F, Grandi AM, Sumner G, Hiralal R, Lonn E. Apical ballooning syndrome or takotsubo cardiomyopathy: a systematic review. Eur Heart J. 2006;27:1523-9.

4. Akashi YJ, Goldstein DS, Barbaro G, Ueyama T. Takotsubo cardiomyopathy: a new form of acute, reversible heart failure. Circulation. 2008;118:2754-62.

5. Bybee KA, Kara T, Prasad A, Lerman A, Barsness GW, Wright RS, et al. Systematic review: transient left ventricular apical ballooning: a syndrome that mimics ST-segment elevation myocardial infarction. Ann Intern Med. 2004;141:858-65.

6. Richard C. Stress-related cardiomyopathies. Ann Intensive Care. 2011;1:39

7. Tsuchihashi K, Ueshima K, Uchida T, Oh-mura N, Kimura K, Owa M, et al. Transient left ventricular apical ballooning without coronary artery stenosis: a novel heart syndrome mimicking acute myocardial infarction. Angina Pectoris-Myocardial Infarction Investigations in Japan. J Am Coll Cardiol. 2001;38:11.

8. Sharkey SW, Lesser JR, Zenovich AG, Maron MS, Lindberg J, Longe $\mathrm{TF}$, et al. Acute and reversible cardiomyopathy provoked by stress in women from the United States. Circulation 2005;111:472.

9. Desmet WJ, Adriaenssens BF, Dens JA. Apical ballooning of the left ventricle: first series in white patients. Heart. 2003;89:1027.

10. Madhavan M, Prasad A. Proposed Mayo Clinic criteria for the diagnosis of Tako-Tsubo cardiomyopathy and long-term prognosis. Herz. 2010;35:240-4

11. Abe $\mathrm{Y}$, Kondo M. Apical ballooning of the left ventricle: a distinct entity? Heart. 2003:89:974-6.

12. Opolski G, Pawlak MM, Roik MF, Kochanowski J, Scisło P, Piatkowski $\mathrm{R}$, et al. Clinical presentation, treatment, and longer-term outcomes in patients with takotsubo cardiomyopathy in the setting of neurological symptoms: a case report. Pol Arch Med Wewn. 2010;120:231-6. 
Maria do Carmo FEVEREIRO, Maria Inês SIMÕES, Fátima LAMPREIA, Isabel MARCÃO, António GODINHO, Vítor LOPES

\section{Miocardiopatia de Takotsubo: Na Origem do Choque Cardiogénico}

Acta Med Port 2015:28:670-673

Publicado pela Acta Médica Portuguesa, a Revista Científica da Ordem dos Médicos

Av. Almirante Gago Coutinho, 151

1749-084 Lisboa, Portugal.

Tel: +351218428215

E-mail: submissao@actamedicaportuguesa.com

www.actamedicaportuguesa.com

ISSN:0870-399X | e-ISSN: 1646-0758

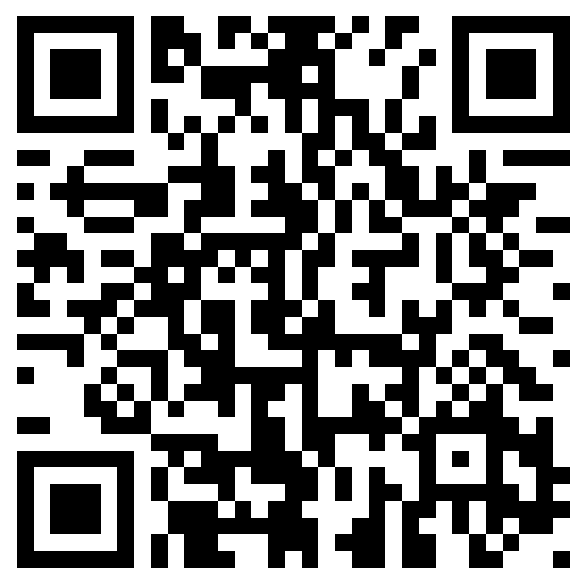

